SU-4252-859

IISc/CHEP $/ 11 / 07$

\title{
Twisted Gauge and Gravity Theories on the Groenewold-Moyal Plane
}

\author{
A. P. Balachandran ${ }^{a *}$, A. Pinzul ${ }^{b \dagger}$, B. A. Qureshi ${ }^{a \ddagger}$ and S. Vaidya ${ }^{c \S}$ \\ ${ }^{a}$ Department of Physics, Syracuse University, Syracuse NY, 13244-1130, USA. \\ ${ }^{b}$ Instituto de Física, Universidade de São Paulo, C.P. 66318, São Paulo, SP, 05315-970, Brazil. \\ ${ }^{c}$ Centre for High Energy Physics, Indian Institute of Science, Bangalore, 560012, India.
}

\begin{abstract}
Recent work $[1,2]$ indicates an approach to the formulation of diffeomorphism invariant quantum field theories (qft's) on the Groenewold-Moyal (GM) plane. In this approach to the qft's, statistics gets twisted and the $S$-matrix in the non-gauge qft's become independent of the noncommutativity parameter $\theta^{\mu \nu}$. Here we show that the noncommutative algebra has a commutative spacetime algebra as a substructure: the Poincaré, diffeomorphism and gauge groups are based on this algebra in the twisted approach as is known already from the earlier work of [1]. It is natural to base covariant derivatives for gauge and gravity fields as well on this algebra. Such an approach will in particular introduce no additional gauge fields as compared to the commutative case and also enable us to treat any gauge group (and not just $U(N)$ ). Then classical gravity and gauge sectors are the same as those for $\theta^{\mu \nu}=0$, but their interactions with matter fields are sensitive to $\theta^{\mu \nu}$. We construct quantum noncommutative gauge theories (for arbitrary gauge groups) by requiring consistency of twisted statistics and gauge invariance. In a subsequent paper (whose results are summarized here), the locality and Lorentz invariance properties of the $S$-matrices of these theories will be analyzed, and new non-trivial effects coming from noncommutativity will be elaborated.

This paper contains further developments of [3] and a new formulation based on its approach.
\end{abstract}

\section{Introduction}

If there is a symmetry group $G$ with elements $g$ and it acts on a single particle Hilbert space $\mathcal{H}$ by the unitary representation $g \rightarrow U(g)$, then conventionally it acts on the two-particle Hilbert space $\mathcal{H} \otimes \mathcal{H}$ by the representation

$$
g \rightarrow U(g) \otimes_{\mathbb{C}} U(g):=\left[U \otimes_{\mathbb{C}} U\right](g \otimes g) .
$$

\footnotetext{
*bal@phy.syr.edu

†apinzul@fma.if.usp.br

${ }^{\ddagger}$ bqureshi@phy.syr.edu

$\S$ vaidya@cts.iisc.ernet.in
} 
(The tensor product of vector spaces hereafter will always be over $\mathbb{C}$.) If it acts on Hilbert spaces $\mathcal{H}_{1}$ and $\mathcal{H}_{2}$ by representations $U_{1}$ and $U_{2}$, then conventionally it acts on $\mathcal{H}_{1} \otimes \mathcal{H}_{2}$ by the representation

$$
g \rightarrow\left[U_{1} \otimes U_{2}\right](g \otimes g)
$$

The homomorphism

$$
\begin{aligned}
\Delta: G & \rightarrow G \otimes G, \\
g & \rightarrow \Delta(g):=g \otimes g
\end{aligned}
$$

underlying (1.2) and (1.3) is said to be a coproduct on $G$. The existence of such a homomorphism is essential for physics. For example, it is the coproduct which determines how a diquark wavefunction transforms under color $S U(3)$, once we agree that each quark transforms by its $\underline{3}$ representation.

Let $G^{*}$ be the group algebra of $G$. If $G$ admits a left- and right-invariant measure $d \mu$, as is generally the case in physics, and $\alpha, \beta: G \rightarrow \mathbb{C}$ are smooth compactly supported functions on $G$, then $G^{*}$ contains the generating elements

$$
\int d \mu(g) \alpha(g) g, \quad \int d \mu\left(g^{\prime}\right) \beta\left(g^{\prime}\right) g^{\prime}
$$

with product

$$
\int d \mu(g) d \mu\left(g^{\prime}\right) \alpha(g) \beta\left(g^{\prime}\right) g g^{\prime}=\int d \mu(g)\left(\alpha *_{c} \beta\right)(g) g
$$

where $\left(\alpha *_{c} \beta\right)(g)$ is the convolution of $\alpha$ and $\beta$ :

$$
\left(\alpha *_{c} \beta\right)(g)=\int d \mu\left(g^{\prime}\right) \alpha\left(g^{\prime}\right) \beta\left(g^{-1} g\right) .
$$

It is necessary to complete the algebra generated by (1.4) in a suitable topology to get all of $G^{*}$.

The coproduct (1.3) extends by linearity as the homomorphism

$$
\begin{aligned}
\Delta: G^{*} & \rightarrow G^{*} \otimes G^{*} \\
\int d \mu(g) \alpha(g) g & \rightarrow \int d \mu(g) \alpha(g) \Delta(g)
\end{aligned}
$$

on $G^{*}$. The representation $U_{i}$ of $G^{*}$ on $\mathcal{H}_{i}$,

$$
U_{i}: \int d \mu(g) \alpha(g) g \rightarrow \int d \mu(g) \alpha(g) U_{i}(g),
$$

induced by those of $G$, also extend to the representation $U_{1} \otimes U_{2}$ on $\mathcal{H}_{1} \otimes \mathcal{H}_{2}$ :

$$
U_{1} \otimes U_{2}: \int d \mu(g) \alpha(g) g \rightarrow \int d \mu(g) \alpha(g)\left[U_{1} \otimes U_{2}\right] \Delta(g)
$$

Next we outline the action of the Poincare group, and more generally of the diffeomorphism group, on the Groenewold-Moyal (GM) plane $\mathcal{A}_{\theta}\left(\mathbb{R}^{N}\right)$. The algebra $\mathcal{A}_{\theta}\left(\mathbb{R}^{N}\right)$ consists of smooth functions on $\mathbb{R}^{N}$ with the multiplication map

$$
\begin{aligned}
m_{\theta}: \mathcal{A}_{\theta}\left(\mathbb{R}^{N}\right) \otimes \mathcal{A}_{\theta}\left(\mathbb{R}^{N}\right) & \rightarrow \mathcal{A}_{\theta}\left(\mathbb{R}^{N}\right), \\
\alpha \otimes \beta & \rightarrow \alpha e^{\frac{i}{2} \overleftarrow{\partial}_{\mu} \theta^{\mu \nu} \vec{\partial}_{\nu}} \beta:=\alpha * \beta
\end{aligned}
$$


where $\theta^{\mu \nu}$ is a constant antisymmetric tensor.

Let

$$
F_{\theta}=e^{\frac{i}{2} \partial_{\mu} \otimes \theta^{\mu \nu}} \partial_{\nu}=\text { "Twist element". }
$$

Then

$$
m_{\theta}(\alpha \otimes \beta)=m_{0}\left[F_{\theta} \alpha \otimes \beta\right]
$$

where $m_{0}$ is the point-wise multiplication map, also defined by (1.10).

Let $\phi$ be an element of the connected component of the diffeomorphism (diffeo) group $\mathcal{D}_{0}\left(\mathbb{R}^{N}\right)$ of $\mathbb{R}^{N}$. The connected component $\mathcal{P}_{+}^{\uparrow}$ of the Poincaré group is a subgroup of $\mathcal{D}_{0}\left(\mathbb{R}^{N}\right)$. For $x \in \mathbb{R}^{N}$,

$$
\phi: x \rightarrow \phi(x) \in \mathbb{R}^{N} .
$$

It acts on functions on $\mathbb{R}^{N}$ by pull-back:

$$
\phi: \alpha \rightarrow \phi^{*} \alpha, \quad\left(\phi^{*} \alpha\right)(x)=\alpha\left[\phi^{-1}(x)\right] .
$$

The work of [1] based on Drinfel'd's basic paper [4] shows that $\mathcal{D}_{0}\left(\mathbb{R}^{N}\right)$ acts on $\mathcal{A}_{\theta}\left(\mathbb{R}^{N}\right)$ compatibly with $m_{\theta}$ if its coproduct is "twisted" to $\Delta_{\theta}$ where

$$
\Delta_{\theta}(\phi)=F_{\theta}^{-1}(\phi \otimes \phi) F_{\theta} .
$$

The right-hand side of (1.15) contains polynomials in derivatives. So it may be best to interpret $\Delta_{\theta}$ in terms of $\mathcal{D}_{0}\left(\mathbb{R}^{N}\right)^{*}$.

We denote the representation of $\phi$ on $\mathcal{A}_{\theta}\left(\mathbb{R}^{N}\right) \otimes \mathcal{A}_{\theta}\left(\mathbb{R}^{N}\right)$ by $\Delta_{\theta}(\phi)$ omitting symbols like $U \otimes U$ which occur in (1.2).

The restriction to the connected component of $\mathcal{D}_{0}\left(\mathbb{R}^{N}\right)$ is not essential. The discussion can be extended to parity and time-reversal [5].

For $\theta^{\mu \nu}=0$ and scalar bosons, statistics is imposed on the two-particle sector by working with the symmetrized tensor product $\mathcal{A}_{0}\left(\mathbb{R}^{N}\right) \otimes_{s} \mathcal{A}_{0}\left(\mathbb{R}^{N}\right)$. It has elements $v \otimes_{s} w$ where

$$
v \otimes_{s} w=\frac{1}{2}[v \otimes w+w \otimes v], \quad v, w \in \mathcal{A}_{0}\left(\mathbb{R}^{N}\right) .
$$

But the twisted coproduct does not preserve symmetrization $[2,4,6]$,

$$
\Delta_{\theta}(\phi)\left(v \otimes_{s} w\right) \notin \mathcal{A}_{0}\left(\mathbb{R}^{N}\right) \otimes_{s} \mathcal{A}_{0}\left(\mathbb{R}^{N}\right)
$$

if $v$ and $w$ are not zero. We are hence obliged to twist statistics as well. Thus let $\tau_{0}$ be the flip map:

$$
\tau_{0}(v \otimes w)=w \otimes v .
$$

Then

$$
\tau_{\theta}:=F_{\theta}^{-1} \tau_{0} F_{\theta}=F_{\theta}^{-2} \tau_{0}
$$

commutes with $\Delta_{\theta}(\phi)$. It is an involution,

$$
\tau_{\theta}^{2}=F_{\theta}^{-1} \tau_{0}^{2} F_{\theta}=\mathbf{1} \otimes \mathbf{1}=\mathrm{id}
$$

and the tensor product $\mathcal{A}_{\theta}\left(\mathbb{R}^{N}\right) \otimes_{s_{\theta}} \mathcal{A}_{\theta}\left(\mathbb{R}^{N}\right)$ with twisted symmetrization consists of elements

$$
v \otimes_{s_{\theta}} w=\frac{1}{2}\left[\mathrm{id}+\tau_{\theta}\right](v \otimes w) .
$$


The space $\mathcal{A}_{\theta}\left(\mathbb{R}^{N}\right) \otimes_{s_{\theta}} \mathcal{A}_{\theta}\left(\mathbb{R}^{N}\right)$ is invariant under the twisted diffeos $\Delta_{\theta}(\phi) \underline{1}$

In a similar way, we can argue that the standard antisymmetrization $\left(\mathbf{1}-\tau_{0}\right)(v \otimes w)$ is incompatible with the twisted coproduct, and that the two-particle sector of the twisted fermions has wavefunctions $v \otimes_{a_{\theta}} w$ in $\frac{1}{2}\left(\mathbf{1}-\tau_{\theta}\right) \mathcal{A}_{\theta}\left(\mathbb{R}^{N}\right) \otimes \mathcal{A}_{\theta}\left(\mathbb{R}^{N}\right)$ :

$$
v \otimes_{a_{\theta}} w=\frac{1}{2}\left(\mathbf{1}-\tau_{\theta}\right)(v \otimes w) .
$$

In standard quantum physics with $\theta^{\mu \nu}=0$, the statistics operator $\tau_{0}$ is superselected: all observables commute with $\tau_{0}$. Following this lead, we assume that such a superselection rule holds also for $\theta^{\mu \nu} \neq 0$, and that all observables commute with $\tau_{\theta}$.

The creation-annihilation operators of quantum fields appropriate to (1.21) and (1.22) have been written down before in terms of operators for $\theta^{\mu \nu}=0$ [2]. They will be recalled later.

In this paper, we will show that there is a representation of the commutative algebra $\mathcal{A}_{0}\left(\mathbb{R}^{N}\right)$ on $\mathcal{A}_{\theta}\left(\mathbb{R}^{N}\right)$. We can construct Poincaré and diffeo generators as certain natural differential operators based on $\mathcal{A}_{0}\left(\mathbb{R}^{N}\right)$. Their exponentiation also gives a representation of the associated groups. It is remarkable that acting on $\mathcal{A}_{\theta}\left(\mathbb{R}^{N}\right)$, their coproduct is precisely $\Delta_{\theta}$. Further considerations of this work are based on this striking fact.

This representation of the Poincaré group on $\mathcal{A}_{\theta}\left(\mathbb{R}^{N}\right)$ is not new. It was first discussed by Calmet [7] and analyzed further in [8]. Their emphasis however differs from ours.

Section 2 constructs the commutative algebra $\mathcal{A}_{0}\left(\mathbb{R}^{N}\right)$ which acts on $\mathcal{A}_{\theta}\left(\mathbb{R}^{N}\right)$. The Poincaré generators $M_{\mu \nu}$ and in fact vector fields $v$ in general act on elements of $\mathcal{A}_{\theta}\left(\mathbb{R}^{N}\right)$ in the standard way for the twisted action as well. Knowing this, we point out that we can write any vector field $v$ (of which $M_{\mu \nu}$ is an example) as $v^{\mu} \partial_{\mu}$ where $v^{\mu} \in \mathcal{A}_{0}\left(\mathbb{R}^{N}\right)$ and $\partial_{\mu}$ are the usual coordinate derivatives.

Section 3 contains the crucial result that the preceding actions of $M_{\mu \nu}$ and $v$ fulfill the deformed Leibnitz rule of [1] which follows from the deformed coproduct.

The deformed coproduct on diffeos is introduced for the purpose of preserving the diffeo invariance of qft's. For $\theta^{\mu \nu}=0$, qft's are invariant under gauge groups $\mathcal{G}$ based on "global groups" $G$ as well, and they are fundamental for basic theory. The Poincaré group $\mathcal{P}$ or the diffeo group $\mathcal{D}\left(\mathbb{R}^{N}\right)$ acts on $\mathcal{G}$ and the group governing a basic theory is the semi-direct product $\mathcal{G} \ltimes \mathcal{P}$ on Minkowski space and $\mathcal{G} \ltimes \mathcal{D}\left(\mathbb{R}^{N}\right)$ for gravity plus matter. Once we decide to preserve $\mathcal{P}$ or $\mathcal{D}\left(\mathbb{R}^{N}\right)$ for $\theta^{\mu \nu} \neq 0$, it is natural to try to preserve also $\mathcal{G} \ltimes \mathcal{P}$ and $\mathcal{G} \ltimes \mathcal{D}\left(\mathbb{R}^{N}\right)$. This is easily done: we just have to identify $\mathcal{G}$ as the group of maps from the commutative coordinates underlying $\mathcal{A}_{0}\left(\mathbb{R}^{N}\right)$ to $G$. The rest of the paper explores the consequences of this identification. Such an identification has been done before by [1]. Our development of field theories is different from theirs.

A summary of our results is as follows. Sections 4 and 5 develop an approach to field theories where gravity and gauge theories without matter are identical to their commutative counterparts for $\theta^{\mu \nu}=0$. Recall that in previous work $[9,10]$, the independence of the $S$ matrix from $\theta^{\mu \nu}$ was established for matter without gauge couplings. But these dual facts about matter and connections do not mean that all effects of $\theta^{\mu \nu}$ disappear. Pauli principle is for example affected $[2,11]$. They are also very much present in the coupling of matter

\footnotetext{
${ }^{1}$ This immediately follows from the observation that twisted coproduct commutes with $\tau_{\theta}: \Delta_{\theta}(g) \tau_{\theta}=$ $F_{\theta}^{-1} \Delta_{0}(g) F_{\theta} F_{\theta}^{-1} \tau_{0} F_{\theta}=\tau_{\theta} \Delta_{\theta}(g)$.
} 
and gauge fields. A clear understanding of the latter requires an elucidation of how gauge transformations act on matter fields, or $\mathcal{A}_{\theta}\left(\mathbb{R}^{N}\right)$ modules, which we do in Sections 6 and 7. In section 8 , we construct quantum noncommutative gauge theories, and show that for a $U(1)$ gauge theory, the scattering operator is the same as the one for usual QED.

New effects arise for non-abelian gauge theories, with the emergence of new types of vertices. The perturbative $S$-matrices of the above processes are not Lorentz invariant despite all our elaborate efforts to preserve it. (However they are unitary, consistently with [12] and contrary to certain claims.) The reasons for this will be elaborated in a subsequent paper [13], where we will discuss the relation between locality and Lorentz invariance of the $S$-matrix (see also [14] in this connection).

It appears that the formulation of field theories on $\mathcal{A}_{\theta}\left(\mathbb{R}^{N}\right)$ is not unique. Thus in particular, even though the Hopf algebras describing the diffeo and gauge groups are identical in our work and that of [15-17], the formulations of gravity and gauge field theories are not the same. But it is possible to describe the connection between the two. We shall briefly do so towards the end of sections 4 and 7 .

This paper is an outgrowth of our previous work [3] and develops a new formulation of gauge theories based on its ideas.

\section{The Commutative Algebra $\mathcal{A}_{0}\left(\mathbb{R}^{N}\right)$}

The algebra $\mathcal{A}_{\theta}\left(\mathbb{R}^{N}\right)$, regarded as a vector space, is a module over $\mathcal{A}_{0}\left(\mathbb{R}^{N}\right)$. We can show this as follows.

For any $\alpha \in \mathcal{A}_{\theta}\left(\mathbb{R}^{N}\right)$, we can define two operators $\hat{\alpha}^{L, R}$ acting on $\mathcal{A}_{\theta}\left(\mathbb{R}^{N}\right)$ :

$$
\hat{\alpha}^{L} \xi=\alpha * \xi, \quad \hat{\alpha}^{R} \xi=\xi * \alpha \quad \text { for } \quad \xi \in \mathcal{A}_{\theta}\left(\mathbb{R}^{N}\right),
$$

where $*$ is the GM product defined by Eq.(1.10) (or, equivalently, by Eq.(1.12)). The maps $\alpha \rightarrow \hat{\alpha}^{L, R}$ have the properties

$$
\begin{aligned}
\hat{\alpha}^{L} \hat{\beta}^{L} & =(\hat{\alpha} \hat{\beta})^{L}, \\
\hat{\alpha}^{R} \hat{\beta}^{R} & =(\hat{\beta} \hat{\alpha})^{R}, \\
{\left[\hat{\alpha}^{L}, \hat{\beta}^{R}\right] } & =0 .
\end{aligned}
$$

The reversal of $\hat{\alpha}, \hat{\beta}$ on the right-hand side of (2.3) means that for position operators,

$$
\left[\hat{x}^{\mu L}, \hat{x}^{\nu L}\right]=i \theta^{\mu \nu}=-\left[\hat{x}^{\mu R}, \hat{x}^{\nu R}\right] .
$$

Hence in view of (2.4),

$$
\hat{x}^{\mu c}=\frac{1}{2}\left(\hat{x}^{\mu L}+\hat{x}^{\mu R}\right)
$$

generates a representation of the commutative algebra $\mathcal{A}_{0}\left(\mathbb{R}^{N}\right)$ :

$$
\left[\hat{x}^{\mu c}, \hat{x}^{\nu c}\right]=0 .
$$

Let $e_{p} \in \mathcal{A}_{\theta}\left(\mathbb{R}^{N}\right)$ be the exponential function for momentum $p$ :

$$
e_{p}(\xi)=e^{-i p \cdot \xi}
$$


Then

$$
\begin{aligned}
\hat{x}^{\mu c} e_{p}(\xi) & =\frac{1}{2}\left(x^{\mu} e^{\frac{i}{2} \overleftarrow{\partial}^{\mu} \theta_{\mu \nu} \vec{\partial}^{\nu}} e_{p}+x^{\mu} \leftrightarrow e_{p}\right)(\xi) \\
& =\xi^{\mu} e^{-i p \cdot \xi}
\end{aligned}
$$

where (2.9) involves point-wise multiplication. Since any $\alpha \in \mathcal{A}_{\theta}\left(\mathbb{R}^{N}\right)$ has the Fourier representation

$$
\alpha=\int d^{N} p \alpha(p) e_{p}
$$

it follows that

$$
\left(\hat{x}^{\mu c} \alpha\right)(\xi)=\xi^{\mu} \alpha(\xi)
$$

and that $\hat{x}^{\mu c}$ generates the commutative algebra $\mathcal{A}_{0}\left(\mathbb{R}^{N}\right)$ acting by point-wise multiplication on $\mathcal{A}_{\theta}\left(\mathbb{R}^{N}\right)$.

This result is implicit in the work of Calmet and coworkers $[7,8]$.

Let us express ad $\hat{x}^{\mu}$ defined by

$$
\hat{x}^{\mu} * \alpha-\alpha * \hat{x}^{\mu}
$$

in terms of the momentum operator $\hat{p}_{\mu}=-i \partial_{\mu}$. This is easily done using the explicit expression for the star-product, Eq.(1.10):

$$
\operatorname{ad} \hat{x}^{\mu} \alpha=x^{\mu} * \alpha-\alpha * x^{\mu}=i \theta^{\mu \nu} \partial_{\nu} \alpha=-\theta^{\mu \nu} \hat{p}_{\nu} .
$$

Hence 2

$$
\hat{x}^{\mu c}=\hat{x}^{\mu L}-\frac{1}{2} \operatorname{ad} \hat{x}^{\mu}=\hat{x}^{\mu L}+\frac{1}{2} \theta^{\mu \nu} \hat{p}_{\nu}
$$

This result is the starting point of the work of Calmet et al $[7,8]$.

The connected Lorentz group $\mathcal{L}_{+}^{\uparrow}$ acts on functions $\alpha \in \mathcal{A}_{\theta}\left(\mathbb{R}^{N}\right)$ in just the usual way in our approach with the coproduct-twist:

$$
[U(\Lambda) \alpha](x)=\alpha\left(\Lambda^{-1} x\right)
$$

for $\Lambda \in \mathcal{L}_{+}^{\uparrow}$ and $U: \Lambda \rightarrow U(\Lambda)$ its representation on functions. Hence the generators $M_{\mu \nu}$ of $\mathcal{L}_{+}^{\uparrow}$ have the representatives

$$
M_{\mu \nu}=\hat{x}_{\mu}^{c} \hat{p}_{\nu}-\hat{x}_{\nu}^{c} \hat{p}_{\mu}, \quad \hat{p}_{\mu}=-i \partial_{\mu}
$$

on $\mathcal{A}_{\theta}\left(\mathbb{R}^{N}\right)$.

Vector fields $v$ are generators of the Lie algebra of the connected component of the diffeomorphism group acting on functions. Just as for $M_{\mu \nu}$, which is a special vector field, we now see that $v$ can be written as

$$
v=v^{\mu}\left(\hat{x}^{c}\right) \partial_{\mu} .
$$

Both (2.16) and (2.17) look like the familiar expressions for $\theta^{\mu \nu}=0$. Nevertheless, their action on $\mathcal{A}_{\theta}\left(\mathbb{R}^{N}\right)$ must involve the twisted coproduct. The next section explains why this is so.

\footnotetext{
${ }^{2}$ If $x^{\mu_{0}}$ is a commutative coordinate in the centre of $\mathcal{A}_{\theta}\left(\mathbb{R}^{N}\right)$, then $\theta^{\mu_{0} \mu}=0, \forall \mu$, and $\hat{x}^{\mu_{0} c} \equiv \hat{x}^{\mu_{0}}$.
} 


\section{On the Twisted Coproduct}

Let us first check the modification of the Leibnitz rule for $M_{\mu \nu}$. We can write, as an identity,

$$
M_{\mu \nu}(\alpha * \beta)=\left(M_{\mu \nu} \alpha\right) * \beta+\alpha *\left(M_{\mu \nu} \beta\right)+\frac{1}{2}\left[\left(\operatorname{ad} \hat{x}_{\mu} \alpha\right) *\left(\hat{p}_{\nu} \beta\right)-\left(\hat{p}_{\nu} \alpha\right) *\left(\operatorname{ad} \hat{x}_{\mu} \beta\right)-\mu \leftrightarrow \nu\right]
$$

which on using (2.13) and the antisymmetry of $\theta^{\mu \nu}$ gives

$$
\begin{aligned}
M_{\mu \nu}(\alpha * \beta) & =\left(M_{\mu \nu} \alpha\right) * \beta+\alpha *\left(M_{\mu \nu} \beta\right) \\
& -\frac{1}{2}\left[\left((\hat{p} \cdot \theta)_{\mu} \alpha\right) *\left(\hat{p}_{\nu} \beta\right)-\left(\hat{p}_{\nu} \alpha\right) *\left((\hat{p} \cdot \theta)_{\mu} \beta\right)-\mu \leftrightarrow \nu\right], \\
(\hat{p} \cdot \theta)_{\rho} & :=\hat{p}_{\lambda} \theta_{\rho}^{\lambda} .
\end{aligned}
$$

Thus the Leibnitz rule is twisted. The twist is exactly what is required by the coproduct $\Delta_{\theta}[19]:$

$$
\begin{aligned}
& \Delta_{\theta}\left(M_{\mu \nu}\right)=\Delta_{0}\left(M_{\mu \nu}\right)-\frac{1}{2}\left[(\hat{p} \cdot \theta)_{\mu} \otimes \hat{p}_{\nu}-\hat{p}_{\nu} \otimes(\hat{p} \cdot \theta)_{\mu}-(\mu \leftrightarrow \nu)\right], \\
& \Delta_{0}\left(M_{\mu \nu}\right)=M_{\mu \nu} \otimes \mathbf{1}+\mathbf{1} \otimes M_{\mu \nu} .
\end{aligned}
$$

Thus

$$
m_{\theta}\left[\Delta_{\theta}\left(M_{\mu \nu}\right) \alpha \otimes \beta\right]=M_{\mu \nu}(\alpha * \beta) .
$$

The operator $M_{\mu \nu}$ is a particular vector field. What we have seen is that it is of the form (2.17). A similar argument shows that all the "twisted" vector fields are of the form (2.17). The connected component of the twisted diffeomorphism group is generated by $v$. It follows that this group is isomorphic to the connected component $\mathcal{D}_{0}\left(\mathbb{R}^{N}\right)$ of the untwisted diffeomorphism group.

\section{Implications for Pure Gravity}

The implications of this observation are striking. We discuss pure gravity first.

Consider the covariant derivative

$$
D_{\mu}=\partial_{\mu}+\Gamma_{\mu}+\omega_{\mu}
$$

where $\Gamma_{\mu}$ and $\omega_{\mu}$ are the Levi-Civita and spin connections respectively.

Under diffeomorphisms, it is natural to assume that $D_{\mu}$ transforms in the usual way. Since the former is generated by vector fields like (2.17), the transformed $D, \Gamma$ and $\omega$ depend on $\hat{x}^{c}$. It is thus natural to assume that just as in the commutative case, $\Gamma$ and $\omega$ depend only on $\hat{x}^{c}$. [But this is an assumption, as the work of [1] which uses an alternative assumption shows (see below)].

Now consider the frame fields $e_{\mu}^{a}$. Just as for $\theta^{\mu \nu}=0$, we can assume that they are covariantly constant,

$$
\partial_{\mu} e_{\nu}^{a}+\Gamma_{\mu \nu}^{\lambda} * e_{\lambda}^{a}+\omega_{\mu b}^{a} * e_{\nu}^{b}=0
$$

and impose also the condition

$$
\Gamma_{\mu \nu}^{\lambda}=\Gamma_{\nu \mu}^{\lambda}
$$


to eliminate torsion. Then (4.2) can be treated just as for $\theta^{\mu \nu}=0$ if we assume that $e_{\mu}^{a}$ depends only on $\hat{x}^{\mu c}$. In that case, the $*$ 's in (4.2) can be erased and $\omega$ can be expressed as

$$
\omega_{\mu b}^{a}=\left(\partial_{\mu} e_{\nu}^{a}\right) e_{b}^{\nu}+\Gamma_{\mu \nu}^{\lambda} e_{\lambda}^{a} e_{b}^{\nu}
$$

We remark that in (4.2), we have the natural freedom to reverse the order of the factors in the last two terms. This ordering ambiguity has no effect for this solution available in our approach, but can be important in other approaches.

We have not studied the possibility of other solutions for (4.3). Perhaps they exist, with $e_{\mu}^{a}$ depending on both $\hat{x}^{\mu c}$ and $\hat{x}^{\mu L}$, but (4.4) is satisfactory and we accept it.

Thus the gravity sector in our approach is based on the commutative coordinate and its algebra is isomorphic (under suitable assumptions) to $\mathcal{A}_{0}\left(\mathbb{R}^{N}\right)$. Hence the gravity sector is based on standard differential geometry. As $\mathcal{A}_{0}\left(\mathbb{R}^{N}\right)$ admits the usual integration, the dynamics in the gravity sector can be described in the manner appropriate for $\theta^{\mu \nu}=0$.

In the formulation of [1], the covariant derivative $D_{\mu}^{*}$ acts with a $*$-product. In their formulation if we use instead

$$
\mathcal{D}_{\mu}^{*}=D_{\mu}^{*} e^{-\frac{i}{2} a d \overleftarrow{\partial}_{\lambda} \theta^{\lambda \rho} \vec{\partial}_{\rho}}
$$

where

$$
D_{\mu}^{*} a d \overleftarrow{\partial}_{\lambda}:=\left[\partial_{\lambda}, D_{\mu}^{*}\right]
$$

as covariant derivative, then

$$
\mathcal{D}_{\mu}^{*} * \alpha=D_{\mu}^{*} \alpha
$$

where there is no $*$ on the right hand side. Hence $\mathcal{D}_{\mu}^{*}$ is our covariant derivative described in their formalism. Both the approaches seem consistent, differing only in the choice of covariant derivative.

\section{Implications for Gauge Fields}

Gauge fields $A_{\lambda}$ transform as one-forms under diffeomorphisms for $\theta^{\mu \nu}=0$. For $\theta^{\mu \nu} \neq 0$, the vector fields $v^{\mu}$ generating diffeomorphisms depend on $\hat{x}^{c}$. If an infinitesimal diffeomorphism acts on $A_{\lambda}$ in a conventional way for $\theta^{\mu \nu} \neq 0$ and $A_{\lambda}$ and its variation $\delta A_{\lambda}$ are to depend on just one combination of noncommutative coordinates, then $A_{\lambda}$ can depend only on $\hat{x}^{c}$. This leads to the conclusion that gauge fields are independent of $\theta^{\mu \nu}$ and are not affected by noncommutativity.

Such an inference is reasonable for another reason as well. Twisted coproducts for diffeomorphisms are introduced to maintain them as symmetries in gravity. But for $\theta^{\mu \nu}=0$, with gravity and gauge fields present, the group of importance is not just $\mathcal{D}_{0}\left(\mathbb{R}^{N}\right)$, but its semi-direct product $\mathcal{G} \ltimes \mathcal{D}_{0}\left(\mathbb{R}^{N}\right)$. Once we decide to maintain $\mathcal{D}_{0}\left(\mathbb{R}^{N}\right)$ as a symmetry group for $\theta^{\mu \nu} \neq 0$, it is natural to go the whole way and preserve $\mathcal{G} \ltimes \mathcal{D}_{0}\left(\mathbb{R}^{N}\right)$ for $\theta^{\mu \nu} \neq 0$. But elements of $\mathcal{D}_{0}\left(\mathbb{R}^{N}\right)$ perform diffeomorphisms, so then we should require that elements of $\mathcal{G}$ are constructed from the elements of the algebra generated by $\hat{x}^{c}$. That would then say that the abstract group $\mathcal{G}$ is independent of $\theta^{\mu \nu}$.

But in our approach $D=d+A$ transforms under $g \in \mathcal{G}$ according to $D \rightarrow g D g^{-1}$. So if $A$ and its gauge transform depend on just one coordinate operator, that operator is $\hat{x}^{c}$. 
If the focus is just on the Poincare group, the above argument is still valid on substituting this group for $\mathcal{D}_{0}\left(\mathbb{R}^{N}\right)$, provided $N \geq 3$. The case $N=2$ is special, since the Poincaré group (in fact the volume preserving diffeomorphism group) with the coproduct $\Delta_{0}$ is an automorphism of $\mathcal{A}_{\theta}\left(\mathbb{R}^{2}\right)$.

The conclusion of the last two sections is that gravity and gauge sectors are unaffected by noncommutativity.

In the standard approach to noncommutative gauge groups [20,21], where covariant derivatives act with the $*$-product, it is possible to treat only particular representations of $U(N)$ gauge groups or use enveloping algebras [22] or deal with the Seiberg-Witten map [23]. (But see Chaichian et. al. [20]). There is no such limitation now where the gauge group is just that for $\theta^{\mu \nu}=0$.

In quantum Hall effect, the algebra of observables is $\mathcal{A}_{\theta}\left(\mathbb{R}^{2}\right) \otimes \mathcal{A}_{\theta}\left(\mathbb{R}^{2}\right)$. In a particular formulation of that system, covariant derivatives of the $U(1)$-gauge fields of electromagnetism do act in the manner we assume, and not with a $*$-product [24].

\section{Gauge Transformations and *-Products}

The Poincaré group was built up from $\hat{x}^{c}$, and not in any other manner, but still its action preserves the $*$-product. We can ask if gauge transformations based on $\hat{x}^{c}$ also preserve the *-product.

\subsection{How the Gauge Group acts on $\mathcal{A}_{\theta}\left(\mathbb{R}^{N}\right)$-Modules}

But this question needs clarification. Fields which transform non-trivially under $\mathcal{G}$ or even the underlying "global" Lie group $G$ are not elements of the algebra $\mathcal{A}_{\theta}\left(\mathbb{R}^{N}\right)$. Rather they are modules over $\mathcal{A}_{\theta}\left(\mathbb{R}^{N}\right)$. If a $d$-dimensional representation of $G$ is involved, they can be elements of $\mathcal{A}_{\theta}\left(\mathbb{R}^{N}\right) \otimes \mathbb{C}^{d}$. They may also be elements of non-trivial projective modules (see for example Chapter 5 of [21]). We focus on $\mathcal{A}_{\theta}\left(\mathbb{R}^{N}\right) \otimes \mathbb{C}^{d}$ for simplicity.

There are two separate matters we have to resolve about these modules. First, we must understand the action of gauge transformations on these modules and show their compatibility with the $*$-product. We argue that we can accomplish such compatibility if the gauge group also has a twisted coproduct. This twist is in fact needed to maintain the semi-direct product structure of $\mathcal{G} \ltimes \mathcal{D}_{0}\left(\mathbb{R}^{N}\right)$ at the level of coproducts. Secondly we must show how to form gauge scalars out of elements of $\mathcal{A}_{\theta}\left(\mathbb{R}^{N}\right) \otimes \mathbb{C}^{d}$ and their adjoints compatibly with the above twisted coproduct. This is an essential step in constructing observables like the Hamiltonian. Below we describe how to accomplish both these tasks successfully. Certain familiar structures available for $\theta^{\mu \nu}=0$ are not available for $\theta^{\mu \nu} \neq 0$. Gauge theories for $\theta^{\mu \nu}=0$ and $\theta^{\mu \nu} \neq 0$ are thus structurally different. The section finally briefly discusses these differences.

The results presented in the section are not new and are due to [1]. So this section can be treated as a review.

Elements $\xi$ of

$$
\mathcal{A}_{\theta}\left(\mathbb{R}^{N}\right)^{d}:=\mathcal{A}_{\theta}\left(\mathbb{R}^{N}\right) \otimes \mathbb{C}^{d}
$$


are $d$-dimensional vectors $\left(\xi_{1}, \xi_{2}, \cdots, \xi_{d}\right)$ where $\xi_{i} \in \mathcal{A}_{\theta}\left(\mathbb{R}^{N}\right)$. There is an action

$$
\begin{aligned}
m_{\theta}: \mathcal{A}_{\theta}\left(\mathbb{R}^{N}\right)^{d} \otimes \mathcal{A}_{\theta}\left(\mathbb{R}^{N}\right) & \rightarrow \mathcal{A}_{\theta}\left(\mathbb{R}^{N}\right)^{d}, \\
\xi \otimes \alpha & \rightarrow m_{\theta}(\xi \otimes \alpha):=\xi * \alpha, \quad \alpha \in \mathcal{A}_{\theta}\left(\mathbb{R}^{N}\right), \\
(\xi * \alpha)_{i} & \equiv \xi_{i} * \alpha,
\end{aligned}
$$

expressing the module property of $\mathcal{A}_{\theta}\left(\mathbb{R}^{N}\right)^{d}$. We treat it as a right-module for convenience.

Now if $g\left(\hat{x}^{c}\right)$ is a $d \times d$ matrix $\in \mathcal{G}$, it transforms $\xi * \alpha$ to $g\left(\hat{x}^{c}\right)(\xi * \alpha)$ where

$$
\left[g\left(\hat{x}^{c}\right)(\xi * \alpha)\right]_{i}(x)=g_{i j}(x)(\xi * \alpha)_{j}(x) .
$$

But when $g_{i j}(x)$ is not a constant,

$$
\text { RHS of }\left(\underline{6.5)} \neq\left(g_{i j}(x) \xi_{j}\right) * \alpha\right. \text {. }
$$

Infinitesimally, for

$$
g\left(\hat{x}^{c}\right) \simeq \mathbf{1}+i \Lambda\left(\hat{x}^{c}\right)
$$

we find from (6.5) that

$$
\Lambda\left(\hat{x}^{c}\right)_{i j}[\xi * \alpha]_{j}=\left(\Lambda_{i j} \xi_{j}\right) * \alpha(x)+\text { extra terms }
$$

which is very much like the deformed Leibnitz rule (3.2).

Let $\epsilon$ be the "counit", the trivial representation of $\mathcal{G}$ :

$$
\epsilon\left(g\left(\hat{x}_{c}\right)\right)=\mathbf{1}
$$

Then

$$
F_{\theta}^{-1}(i d \otimes \epsilon)\left[g\left(\hat{x}^{c}\right) \otimes g\left(\hat{x}^{c}\right)\right] F_{\theta}
$$

acts on $\mathcal{A}_{\theta}\left(\mathbb{R}^{N}\right)^{d} \otimes \mathcal{A}_{\theta}\left(\mathbb{R}^{N}\right)$ according to

$$
\xi \otimes \alpha \rightarrow F_{\theta}^{-1}\left[g\left(\hat{x}^{c}\right) \otimes \mathbf{1}\right] F_{\theta}(\xi \otimes \alpha),
$$

which under $m_{\theta}$ becomes

$$
g\left(\hat{x}^{c}\right)[\xi * \alpha]
$$

which in component form is (6.5).

We thus see that just as the coproduct on diffeos, the twisted coproduct on $\mathcal{G}$,

$$
\Delta_{\theta}\left(g\left(\hat{x}^{c}\right)=F_{\theta}^{-1}\left[g\left(\hat{x}^{c}\right) \otimes g\left(\hat{x}^{c}\right)\right] F_{\theta},\right.
$$

is compatible with the $*$-multiplication in (6.3).

We need this twisted coproduct in any case in order that $\Delta_{\theta}(\phi)$ [cf. (1.15)] acts on $\Delta_{\theta}\left(g\left(\hat{x}^{c}\right)\right.$ compatibly with the semi-direct product structure $\mathcal{G} \ltimes \mathcal{D}_{0}\left(\mathbb{R}^{N}\right)$. 


\subsection{On Gauge Scalars}

If $\eta \in \mathcal{A}_{\theta}\left(\mathbb{R}^{N}\right)^{d}$, and it transforms under $g\left(\hat{x}^{c}\right) \in \mathcal{G}$ according to

$$
\eta(x) \rightarrow\left[g\left(\hat{x}^{c}\right) \eta\right](x)=\left(g_{i j}\left(\hat{x}^{c}\right) \eta_{j}\right)(x)=g_{i j}(x) \eta_{j}(x),
$$

then $\eta^{\dagger}$ necessarily transforms as

$$
\eta^{\dagger} \rightarrow(g \eta)^{\dagger}, \quad \eta_{i}^{\dagger}(x) \rightarrow \eta_{j}^{\dagger}(x) g_{j i}^{*}\left(\hat{x}^{c}\right)=\eta_{j}^{\dagger}(x) g_{j i}^{*}(x)
$$

If $\xi$ and $\xi^{\dagger}$ form another such pair, consider $\sum_{i} \xi_{i}^{*} * \eta_{i} \equiv \xi^{\dagger} * \eta$. It is not invariant if $\xi^{\dagger}$ and $\eta$ are naively transformed as in (6.14) and (6.15). But we want its invariance only for the twisted coproduct (6.13). To check if this is so, we define the "multiplication" map

$$
\delta_{\theta}: \xi^{\dagger} \otimes \eta \rightarrow \xi^{\dagger} * \eta=\delta_{0}\left(F_{\theta} \xi^{\dagger} \otimes \eta\right) .
$$

The representation of $g\left(\hat{x}^{c}\right)$ on $\xi^{\dagger}$ can be denoted by $\overline{i d}$, that on $\eta$ being $i d$. Then

$$
\delta_{\theta}\left[F_{\theta}^{-1}(\overline{i d} \otimes i d)\left(g\left(\hat{x}^{c}\right) \otimes g\left(\hat{x}^{c}\right)\right) F_{\theta} \xi^{\dagger} \otimes \eta\right]=\xi^{\dagger} * \eta
$$

showing its invariance.

\subsection{Transformations of Composite Operators}

For $\theta^{\mu \nu}=0$, if $\psi$ and $\chi$ transform by a gauge group $\mathcal{G}$ as dictated by the representations $\rho$ and $\sigma$ of its global group $G$,

$$
\begin{aligned}
\psi(x) & \rightarrow \rho[g(x)] \psi(x), \quad \chi(x) \rightarrow \sigma[g(x)] \chi(x), \quad g \in G, g(x) \in \mathcal{G}, \\
\psi_{i}(x) & \rightarrow \rho[g(x)]_{i j} \psi_{j}(x), \quad \chi_{\alpha}(x) \rightarrow \sigma[g(x)]_{\alpha \beta} \chi_{\beta}(x),
\end{aligned}
$$

we can consistently assign a transformation law under $\mathcal{G}$ to $\psi \otimes^{\prime} \chi$,

$$
\left(\psi \otimes^{\prime} \chi\right)_{i \alpha}(x, x) \equiv \psi_{i}(x) \chi_{\alpha}(x) .
$$

It is dictated by the representation $\rho \otimes \sigma$ of $G$ :

$$
\begin{aligned}
{\left[\psi \otimes^{\prime} \chi\right]_{i \alpha}(x) } & \rightarrow \rho[g(x)]_{i i^{\prime}} \psi_{i^{\prime}}(x) \sigma[g(x)]_{\alpha \alpha^{\prime}} \chi_{\alpha^{\prime}}(x) \\
& =\rho[g(x)]_{i i^{\prime}} \sigma[g(x)]_{\alpha \alpha^{\prime}} \phi_{i^{\prime}}(x) \chi_{\alpha^{\prime}}(x)
\end{aligned}
$$

In the passage from (6.21) to (6.22), commutativity of spacetime algebra has been used.

We use equations such as (6.22) in forming gauge invariants such as the Yukawa term in the Lagrangian density. It is used as well to form covariant composite local fields such as a color $\overline{3}$ composite of two quark fields.

$\psi \otimes^{\prime} \chi$ is not the tensor product $\psi \otimes \chi$ of $\psi$ and $\chi \cdot \psi \otimes \chi$ is a function on $\mathbb{R}^{N} \otimes \mathbb{R}^{N}$ with value $\psi(x) \otimes \chi(y)$ at $(x, y)$ whereas $\psi \otimes^{\prime} \chi$ is a function of just $(x, x)$, that is, $x$.

We can interpret this restriction in two different ways:

a) $\psi \otimes^{\prime} \chi$ is the restriction of $\psi \otimes \chi$ to the diagonals $(x, x)$.

b) $\left(\psi \otimes^{\prime} \chi\right)_{i \alpha}$ is the product in the algebra, being the $*$-product $\psi_{i} * \chi_{\alpha}$ if $\theta^{\mu \nu} \neq 0$. 
For $\theta^{\mu \nu} \neq 0$ these two interpretations have different implications, although for $\theta^{\mu \nu}=0$, they coincide. Only b) is suitable for $\theta^{\mu \nu} \neq 0$ as we will now argue.

a) Restriction to diagonals: For $\theta^{\mu \nu} \neq 0, \mathcal{G}$ acts on $\psi \otimes \chi$ by the coproduct (6.13). But this action is not compatible with the restriction to $(x, x)$. We can see this in the following way:

$$
\begin{aligned}
& (\psi \otimes \chi)(x, y) \rightarrow(\rho \otimes \sigma) F_{\theta}^{-1}\left[g\left(\hat{x}^{c}\right) \otimes g\left(\hat{x}^{c}\right)\right] F_{\theta}(\psi \otimes \chi)(x, y) \\
= & \exp \left(-\frac{i}{2} \frac{\partial}{\partial x} \wedge \frac{\partial}{\partial y}\right)\left(\rho\left[g\left(\hat{x}^{c}\right)\right] \otimes \sigma\left[g\left(\hat{y}^{c}\right)\right]\right) \exp \left(\frac{i}{2} \frac{\partial}{\partial x} \wedge \frac{\partial}{\partial y}\right)(\psi \otimes \chi)(x, y)
\end{aligned}
$$

where $\frac{\partial}{\partial x} \wedge \frac{\partial}{\partial y}:=\theta^{\mu \nu} \frac{\partial}{\partial x^{\mu}} \otimes \frac{\partial}{\partial y^{\nu}}$.

This is complicated at $x=y$ and involves derivatives of gauge transformations. Its components do not reduce to the analog

$$
\left(\rho\left[g\left(\hat{x}^{c}\right)\right]_{i i^{\prime}} \otimes \sigma\left[g\left(\hat{x}^{c}\right)\right]_{\alpha \alpha^{\prime}}\right)\left(\psi_{i^{\prime}} \otimes \chi_{\alpha^{\prime}}\right)(x, x)
$$

of (6.22). So $\left(\psi \otimes^{\prime} \chi\right)(x, x)$ has no simple transformation law under $\mathcal{G}$.

b) The *-product: In this case the transformation of $\psi \otimes^{\prime} \chi$ is given by

$$
m_{\theta}\left\{(\rho \otimes \sigma) \Delta_{\theta}(g) \psi \otimes \chi\right\}_{i \alpha},
$$

where

$$
(\xi \otimes \eta)(x, y):=\xi_{i}(x) \eta(y)_{\alpha}
$$

To simplify (6.26), we write the twist element $F_{\theta}$ (defined in (1.11) ) in the Sweedler notation:

$$
F_{\theta}=e^{\frac{i}{2} \partial_{\mu} \otimes \theta^{\mu \nu} \partial_{\nu}}=\sum_{n=0}^{\infty} \frac{(i / 2)^{n}}{n !} \theta^{\mu_{1} \nu_{1}} \ldots \theta^{\mu_{n} \nu_{n}} \partial_{\mu_{1}} \ldots \partial_{\mu_{n}} \otimes \partial_{\nu_{1}} \ldots \partial_{\nu_{n}} \equiv \sum_{\gamma} f^{(1) \gamma} \otimes f_{\gamma}^{(2)}
$$

Then (6.26) is

$$
m_{\theta}\left\{(\rho \otimes \sigma) \Delta_{\theta}(g) \psi \otimes \chi\right\}_{i \alpha}=\sum_{\gamma, j, \beta}\left\{\rho\left(g\left(\hat{x}^{c}\right)\right)_{i j} f^{(1) \gamma} \psi_{j}\right\}\left\{\sigma\left(g\left(\hat{x}^{c}\right)\right)_{\alpha \beta} f_{\gamma}^{(2)} \chi_{\beta}(x)\right\} .
$$

As there is no $*$ in (6.29) and the gauge transformations are as for $\theta^{\mu \nu}=0$,

$$
\begin{aligned}
m_{\theta}\left\{(\rho \otimes \sigma) \Delta_{\theta}(g) \psi \otimes \chi\right\}_{i \alpha} & =\sum_{\gamma, j, \beta}\left\{\rho\left(g\left(\hat{x}^{c}\right)\right)_{i j} f^{(1) \gamma} \psi_{j}\right\}\left\{\sigma\left(g\left(\hat{x}^{c}\right)\right)_{\alpha \beta} f_{\gamma}^{(2)} \chi_{\beta}(x)\right\} \\
& =\rho\left[g\left(\hat{x}^{c}\right)\right]_{i j} \sigma\left[g\left(\hat{x}^{c}\right)\right]_{\alpha \beta}\left(\psi_{j} * \chi_{\beta}\right)(x)
\end{aligned}
$$

This is similar to (6.22) so that composite gauge transformations can be consistently defined.

\section{On Covariant Derivatives of Quantum Fields}

Suppose we have a charged scalar field $\phi$,

$$
\phi(x)=\int d \mu(p)\left(a_{p} e^{-i p \cdot x}+b^{\dagger}(p) e^{i p \cdot x}\right)
$$


that obeys twisted statistics in Fock space:

$$
\begin{aligned}
a(p) a(q) & =e^{i p \wedge q} a(q) a(p), \\
a(p) a^{\dagger}(q) & =e^{-i p \wedge q} a^{\dagger}(q) a(p)+2 p_{0} \delta^{(3)}(p-q), \\
b(p) b(q) & =e^{i p \wedge q} b(q) b(p), \\
b(p) b^{\dagger}(q) & =e^{-i p \wedge q} b^{\dagger}(q) b(p)+2 p_{0} \delta^{(3)}(p-q), \\
a(p) b(q) & =e^{i p \wedge q} b(q) a(p), \\
a(p) b^{\dagger}(q) & =e^{-i p \wedge q} b^{\dagger}(q) a(p), \\
a^{\dagger}(p) b^{\dagger}(q) & =e^{i p \wedge q} b^{\dagger}(q) a^{\dagger}(p) .
\end{aligned}
$$

As shown elsewhere $[9,25]$, these relations are direct consequences of the twisted statistics of the multiparticle states discussed in Section 1.

Now the twisted operators $a(p), a^{\dagger}(p), b(p)$ and $b^{\dagger}(p)$ can be realized in terms of untwisted Fock space operators $c(p), d(p)$ as

$$
\begin{aligned}
a(p) & =c(p) e^{-\frac{i}{2} p \wedge P}, \quad a^{\dagger}(p)=c^{\dagger}(q) e^{\frac{i}{2} p \wedge P}, \text { where } \\
P_{\mu} & =\int d \mu(q) q_{\mu}\left[a^{\dagger}(q) a(q)+b^{\dagger}(q) b(q)\right]=\text { the total momentum operator. }
\end{aligned}
$$

Then $\phi(x)$ may be written in terms of the ordinary or commutative fields $\phi_{c}$ as

$$
\phi(x)=\phi_{c} e^{\frac{1}{2} \overleftarrow{\partial} \wedge P}(x)
$$

If $\phi^{\prime}$ is another such quantum field, $\phi^{\prime}(x)=\phi_{c}^{\prime} e^{\frac{1}{2} \overleftarrow{\partial} \wedge P}(x)$, then

$$
\left(\phi * \phi^{\prime}\right)(x)=\left(\phi_{c} \phi_{c}^{\prime}\right) e^{\frac{1}{2} \overleftarrow{\partial} \wedge P}(x)
$$

(17.9, 17.11) are the "dressing transformations" of Grosse, Zamolodchikov and Faddeev [26$28]$.

To define the desirable properties of covariant derivatives $D_{\mu}$, let us first look at ways of multiplying the field $\phi$ by function $\alpha_{c} \in \mathcal{A}_{0}\left(\mathbb{R}^{4}\right)$. There are two possibilities:

$$
\begin{aligned}
& \phi \quad \rightarrow\left(\phi_{c} \alpha_{c}\right) e^{\frac{1}{2} \overleftarrow{\partial} \wedge P} \equiv T_{0}\left(\alpha_{c}\right) \phi \\
& \phi \quad \rightarrow\left(\phi_{c} *_{\theta} \alpha_{c}\right) e^{\frac{1}{2} \overleftarrow{\partial} \wedge P} \equiv T_{\theta}\left(\alpha_{c}\right) \phi
\end{aligned}
$$

In (7.13), $T_{0}$ gives a representation of the commutative algebra of functions, whereas $T_{\theta}$ in (7.14) gives that of the $*$-algebra.

For $D_{\mu}$ to qualify as the covariant derivative of a quantum field associated with $\mathcal{A}_{0}\left(\mathbb{R}^{4}\right)$, we require of it that

1

$$
D_{\mu}\left(T_{0}\left(\alpha_{c}\right) \phi\right)=T_{0}\left(\alpha_{c}\right)\left(D_{\mu} \phi\right)+T_{0}\left(\partial_{\mu} \alpha_{c}\right) \phi
$$

$2 D_{\mu}$ preserve statistics.

$3 D_{\mu}$ preserve Poincaré and gauge invariance. 
The requirement (7.15) reflects the fact that $D_{\mu}$ is associated with the commutative algebra $\mathcal{A}_{0}\left(\mathbb{R}^{4}\right)$.

There are two immediate choices for $D_{\mu} \phi$ :

$$
\begin{aligned}
& \text { 1. } D_{\mu} \phi=\left(\left(D_{\mu}\right)_{c} \phi_{c}\right) e^{\frac{1}{2} \overleftarrow{\partial} \wedge P} \\
& \text { 2. } D_{\mu} \phi=\left(\left(D_{\mu}\right)_{c} e^{\frac{1}{2} \overleftarrow{\partial} \wedge P}\right)\left(\phi_{c} e^{\frac{1}{2} \overleftarrow{\partial} \wedge P}\right)
\end{aligned}
$$

where

$$
\left(D_{\mu}\right)_{c}=\partial_{\mu}+\left(A_{\mu}\right)_{c}
$$

and $\left(A_{\mu}\right)_{c}$ is the commutative gauge field, a function only of $\hat{x}^{c}$. It is easy to see that the second choice does not satisfy (7.15), but the first one does. The first choice is also good because it preserves statistics, Poincaré and gauge invariance.

As regards gauge invariance, we can see it as follows. The generators of gauge transformations are the same as those for $\theta^{\mu \nu}=0$. If we consider $D_{\mu} \phi|0\rangle$, it is the same as the action of $\left(D_{\mu}\right)_{c} \phi_{c}$ on the Fock vacuum. Hence it transforms correctly.

To see the compatibility of gauge transformations and statistics, let us look at the operator product $\left(D_{\mu} \phi\right)(x)\left(D_{\nu} \phi\right)(y)$ and restrict it to the two-particle sector. It reads

$$
\left.\left(D_{\mu}\right)_{c} \phi_{c}(x) e^{-\frac{i}{2} \overleftarrow{\partial} \wedge \vec{\partial}}\left(D_{\mu}\right)_{c} \phi_{c}(y)\right)|0\rangle
$$

The Gauss law operator only transforms the operator parts of $\left(D_{\mu}\right)_{c} \phi_{c}$ which are the analogs of creation-annihilation operators $a^{\dagger}(p), a(p)$. That is, if

$$
\left(D_{\mu}\right)_{c} \phi_{c}(x)=\sum_{n} \alpha_{\mu}^{n} f_{n}(x)
$$

then Gauss law only transforms the operators $\alpha_{\mu}^{n}$. So under gauge transformations $g$,

$$
\left(D_{\mu}\right)_{c} \phi_{c}(x) e^{-\frac{i}{2} \overleftarrow{\partial} \wedge \vec{\partial}}\left(D_{\mu}\right)_{c} \phi_{c}(y)|0\rangle \rightarrow\left(g \alpha_{\mu}^{n}\right)\left(g \alpha_{\nu}^{m}\right)\left(f_{n}(x) e^{-\frac{i}{2} \overleftarrow{\partial} \wedge \vec{\partial}} f_{m}(y)\right)|0\rangle
$$

Under the multiplication map, the exponential cancels out, and

$$
m_{\theta}\left(\left(g \alpha_{\mu}^{n}\right)\left(g \alpha_{\nu}^{m}\right)\left(f_{n}(x) e^{-\frac{i}{2} \overleftarrow{\partial}_{x} \wedge \vec{\partial}_{y}} f_{m}(y)\right)\right)|0\rangle=g\left[\left(D_{\mu}\right)_{c} \phi_{c}\right] g\left[\left(D_{\nu}\right)_{c} \phi_{c}\right]|0\rangle
$$

Note that since the symmetry generators are the same as those for $\theta^{\mu \nu}=0$, so the $\left(F_{\mu \nu}\right)^{2}$ term of the gauge field interaction also transforms correctly.

Any gauge group can be treated in this approach, unlike some other approaches.

Similar arguments can be made about the transformation properties under the Poincaré group.

The relation between our covariant derivative and that of [1] is similar to the relation between the corresponding operators appropriate for diffeos. We previously described this connection in Section 4 . Thus the gauge covariant derivative $D_{\mu}$ of [1] acts with a $*$-product on fields. Consider a new covariant derivative

$$
\mathcal{D}_{\mu}=D_{\mu} e^{-\frac{i}{2} a d \overleftarrow{\partial}_{\lambda} \theta^{\lambda \rho} \vec{\partial}_{\rho}}
$$

acting with a $*$-product on fields $\alpha$. This action becomes our action of $D_{\mu}$ on $\alpha$ :

$$
\mathcal{D}_{\mu} * \alpha=D_{\mu} \alpha .
$$

The relation between the two covariant derivatives can be understood in this manner. 


\section{Quantum Gauge Theory}

Having identified the correct covariant derivative, it is simple to write down the Hamiltonian for gauge theories. The commutator of two covariant derivatives gives us the curvature. Using (17.16)

$$
\begin{aligned}
{\left[D_{\mu}, D_{\nu}\right] \phi } & =\left(\left[D_{\mu c}, D_{\nu, c}\right] \phi_{c}\right) e^{\frac{1}{2} \overleftarrow{\partial} \wedge P} \\
& =\left(F_{\mu \nu, c} \phi_{c}\right) e^{\frac{1}{2} \overleftarrow{\partial} \wedge P}
\end{aligned}
$$

As $F_{\mu \nu, c}$ above transforms covariantly under gauge transformations, we can use it to construct the Hamiltonian for the gauge theory. Thus pure gauge theories on the GM plane are identical to their counterparts on commutative space.

However, the coupling between matter and gauge field, which involves the covariant derivative of the matter field, is different from its commutative analog. As a result, the interaction Hamiltonian splits into two parts:

$$
\begin{aligned}
H_{\theta}^{I}=\int d^{3} x\left[\mathcal{H}_{\theta}^{M G}+\mathcal{H}_{\theta}^{G}\right], \quad M G & =\text { matter - gauge, } \quad G=\text { pure gauge field } \\
\mathcal{H}_{\theta}^{M G} & =\mathcal{H}_{0}^{M G} e^{\frac{1}{2} \overleftarrow{\partial} \wedge P}, \\
\mathcal{H}_{\theta}^{G} & =\mathcal{H}_{0}^{G} .
\end{aligned}
$$

We include matter-gauge field couplings and all matter couplings in $\mathcal{H}_{\theta}^{M G}$, while $\mathcal{H}_{\theta}^{G}$ contains only gauge field terms. For QED, $\mathcal{H}_{\theta}^{G}=0$, so as shown in [9], the $S$-operator of the theory is the same as the commutative case:

$$
S_{\theta}^{Q E D}=S_{0}^{Q E D}
$$

For the Standard Model $(\mathrm{SM}), \mathcal{H}_{\theta}^{G}=\mathcal{H}_{0}^{G} \neq 0$. As this term has no statistics twist,

$$
S_{\theta}^{S M} \neq S_{0}^{S M} \text {. }
$$

because of the cross-terms in the $S$-matrix between $\mathcal{H}_{\theta}^{M G}$ and $\mathcal{H}_{\theta}^{G}$. In particular, this inequality happens in QCD. Processes like $q g \rightarrow q g$ via a gluon exchange interaction actually also violate causality and Lorentz invariance, as we indicate below. 3

The Feynman diagram responsible for this violation is shown in Fig 1. The twist of $\mathcal{H}_{0}^{M G}$ in (8.4) changes the gluon propagator that connects the quark-quark-gluon vertex to the 3gluon vertex (and in fact to any vertex containing just gluons). This propagator is different from the usual one by its dependence on terms of the form $\overrightarrow{\theta^{0}} \cdot \vec{P}_{\text {inc }}$, where $\left(\overrightarrow{\theta^{0}}\right)_{i}=\theta^{0 i}$ and $\vec{P}_{i n c}$ is the total momentum of the incoming particles. Such dependence is clearly framedependent and violates Lorentz invariance (Their $C, P$, and $T$ properties are discussed in $[5])$.

Acknowledgments: It is a pleasure to thank Earnest Akofor, T. R. Govindarajan, Sang Jo and Anosh Joseph for discussions. APB especially thanks Paolo Aschieri for a clarifying discussion about Eq (7.13 - 7.15). Some of the results of this papers overlap with some of those of [5]. The work of APB and BQ is supported in part by DOE under grant number DE-FG02-85ER40231. The work of AP has been supported by FAPESP grant number 06/56056-0.

\footnotetext{
${ }^{3} \mathrm{~A}$ more in-depth discussion of causality in noncommutative theories will be presented elsewhere [13].
} 


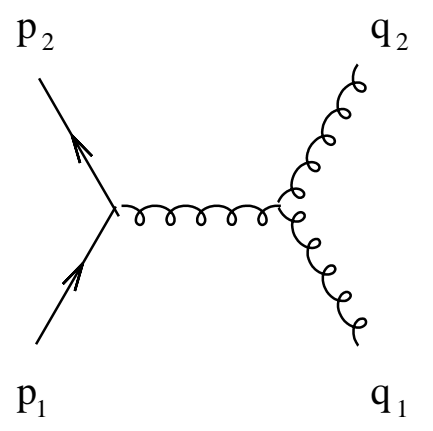

Figure 1: A Feynman diagram with a non-trivial $\theta$-dependence

\section{References}

[1] P. Aschieri, C. Blohmann, M. Dimitrijevic, F. Meyer, P. Schupp and J. Wess, Class. Quant. Grav. 22, 3511 (2005); arXiv:hep-th/0504183.

[2] A.P. Balachandran, G. Mangano, A. Pinzul, S. Vaidya, Int. J. Mod. Phys. A 21, 3111 (2006); arXiv:hep-th/0508002.

[3] A.P. Balachandran, A. Pinzul, B. Qureshi and S. Vaidya, arXiv:hep-th/0608138].

[4] V. G. Drinfel'd, Leningrad Math. J. 1, 1419-1457, (1990).

[5] E. Akofor, A. P. Balachandran, S. G. Jo and A. Joseph, arXiv:0706.1259 [hep-th] (to appear in JHEP).

[6] R. Oeckl, Nucl. Phys. B 581, 559 (2000) arXiv:hep-th/0003018.

[7] X. Calmet, Phys. Rev. D 71, 085012 (2005) arXiv:hep-th/0411147.

[8] X. Calmet and A. Kobakhidze, Phys. Rev. D 72, 045010 (2005) [arXiv:hep-th/0506157].

[9] A. P. Balachandran, A. Pinzul and B. Qureshi, Phys. Lett. B 634, 434 (2006); arXiv:hep-th/0508151].

[10] A. Pinzul, Int. J. Mod. Phys. A 20, 6268 (2005).

[11] B. Chakraborty, S. Gangopadhyay, A. G. Hazra and F. G. Scholtz, J. Phys. A 39, 9557 (2006); arXiv:hep-th/0601121.

[12] D. Bahns, S. Doplicher, K. Fredenhagen and G. Piacitelli, Phys. Lett. B 533, 178 (2002) arXiv:hep-th/0201222.

[13] A.P. Balachandran, A. Pinzul, B. Qureshi and S. Vaidya, arXix:0708.1379 [hep-th].

[14] H. Grosse and G. Lechner, arXiv:0706.3992 [hep-th].

[15] D. V. Vassilevich, Mod. Phys. Lett. A 21, 1279 (2006); arXiv:hep-th/0602185]. 
[16] P. Aschieri, M. Dimitrijevic, F. Meyer, S. Schraml and J. Wess, Lett. Math. Phys. 78, 61 (2006); arXiv:hep-th/0603024.

[17] A. Kobakhidze, arXiv:hep-th/0603132].

[18] A. P. Balachandran, A. Pinzul and B. Qureshi, arXiv:0708.1779 [hep-th].

[19] M. Chaichian, P. P. Kulish, K. Nishijima and A. Tureanu, Phys. Lett.B 604, 98 (2004); arXiv:hep-th/0408069].

[20] M. Chaichian, P. Presnajder, M. M. Sheikh-Jabbari and A. Tureanu, Phys. Lett. B 526, 132 (2002) arXiv:hep-th/0107037.

[21] A.P. Balachandran, S. Kurkcuoglu and S. Vaidya, Lectures on Fuzzy and Fuzzy SUSY Physics, World Scientific, Singapore, 2007.

[22] B. Jurco, S. Schraml, P. Schupp and J. Wess, Eur. Phys. J. C 17, 521 (2000) arXiv:hep-th/0006246.

[23] N. Seiberg and E. Witten, JHEP 9909, 032 (1999) arXiv:hep-th/9908142.

[24] A. P. Balachandran, K. S. Gupta and S. Kurkcuoglu, arXiv:0707.1219 [hep-th].

[25] A. P. Balachandran, T. R. Govindarajan, G. Mangano, A. Pinzul, B. A. Qureshi and S. Vaidya, Phys. Rev. D 75, 045009 (2007); arXiv:hep-th/0608179.

[26] H. Grosse, Phys. Lett. B 86, 267 (1979).

[27] A. B. Zamolodchikov and Al. B. Zamolodchikov, Annals Phys. 120, 253 (1979).

[28] L. Faddeev, Sov. Sci. Rev. C 1, 107 (1980). 